\title{
Organic Brain Syndrome
}

National Cancer Institute

\section{Source}

National Cancer Institute. Organic Brain Syndrome. NCI Thesaurus. Code C34868.

A mental disorder caused by intrinsic disease which is characterized by acute or chronic impairment of intellectual functioning, behavior, mood or judgment. It is attributable to a medical etiology and excludes a primary psychiatric cause. A chronic course usually correlates to a poorer prognosis. 